\title{
Determination of the amorphous phase in titania and its influence on photocatalytic properties
}

\author{
Guang $\mathrm{HU}^{\mathrm{a}}$, Sainan CHEN, Qinfang SHI, Xiang HE, Pengcheng CHEN \\ Department of Physics, Huaiyin Institute of Technology, Huaian 223003, P. R. China \\ aemail: hygxyhg@126.com
}

Keywords: Amorphous; Mesoporous; Photocatalytic

\begin{abstract}
In the present work, we compare the photocatalytic activity of titania samples normalized to the sample weight and specific surface area (SSA), and show a correlation between the PCA of titania catalysts with similar phase compositions and amorphous phase contents. Catalysts with a significant amorphous content were annealed and processed by partial dissolution in acid and included in the analysis. PCA was measured by methyl orange (MO) photodegradation in an aqueous catalyst suspension under high-pressure $\mathrm{Hg}$ bulb illumination and the $\mathrm{pH}$ was controlled using phosphate buffer ( $\mathrm{pH}$ 6.9). The weight percentage of the amorphous phase was determined using reference intensity ratios (RIR) for X-ray diffraction patterns of the titania samples measured with crystalline silicon additives. The reproducibility of the proposed method was demonstrated by measuring the amorphous content in mixtures of the sample and XRD-amorphous titania. The obtained results show that the PCA of the titania samples decreases with the weight percentage of the amorphous phase.
\end{abstract}

\section{Introduction}

Due to its photochemical properties, titania are extensively studied in many practical applications, such as water photolysis, solar cell, etc. [1]. The following characteristics of titanium dioxide are usually considered key factors that affect high photocatalytic activity (PCA): high surface area, surface hydroxyls, crystallinity, and optimal particle size. High surface area is believed to have more active site for photocatalytic reaction, on the other hand, high surface is usually associated with low crystallinity and a high concentration of defects, and these may lead to low photocatalytic activity [2] [3]. The amorphous phase means high concentration of structural defects, which has no photocatalytic activity. Thus, comparing the PCA of different samples, we must determine the amorphous phase content [4] [5]. But analyzing the amorphous phase is limited for the complicated atomic structure. The aim of this essay was to develop a method for quantitative analysis of the amorphous phase content in $\mathrm{TiO}_{2}$ and to determine the impact of amorphous phase on PCA.

\section{Experimental}

Mesoporous titania was synthesized with a solvothermal method [6]. 2g Pluronic P123 (molar weight $5800 \mathrm{~g} / \mathrm{mol}$, Aldrich 435465) was dissolved into $60 \mathrm{ml} \mathrm{HCl}$ solution ( $\mathrm{pH}=2$, Aldrich 38282), then added $17 \mathrm{mmol}$ tetrabutyl titanate (TBT) in the solution. The solution was kept in a $80^{\circ} \mathrm{C}$ oven 24h. The result product was washed with deionized water and ethanol three times and dried in an oven. The resultant powders were calcined at $360^{\circ} \mathrm{C}$ tube furnace for 12 hours and produce the final mesoporous $\mathrm{TiO}_{2}$. Commercial titania samples, P25 and Hombikat UV100 were used without modification.

\section{Results and discussion}

The sample microstructure was characterized using scanning electron microscopy (SEM, Zeiss Leo Supra 50VP) and transmission electron microscopy (TEM, Zeiss Libra 200). Figure 1a shows scanning electron microscopy (SEM) images of the prepared calcined mesoporous $\mathrm{TiO}_{2}$ using Zeiss 
Leo Supra 50VP. The samples possessed very smooth surfaces with a diameter of 1200nm. In TEM images, there are micropore surrounded around the surface, and the diameter is about $4 \mathrm{~nm}$.
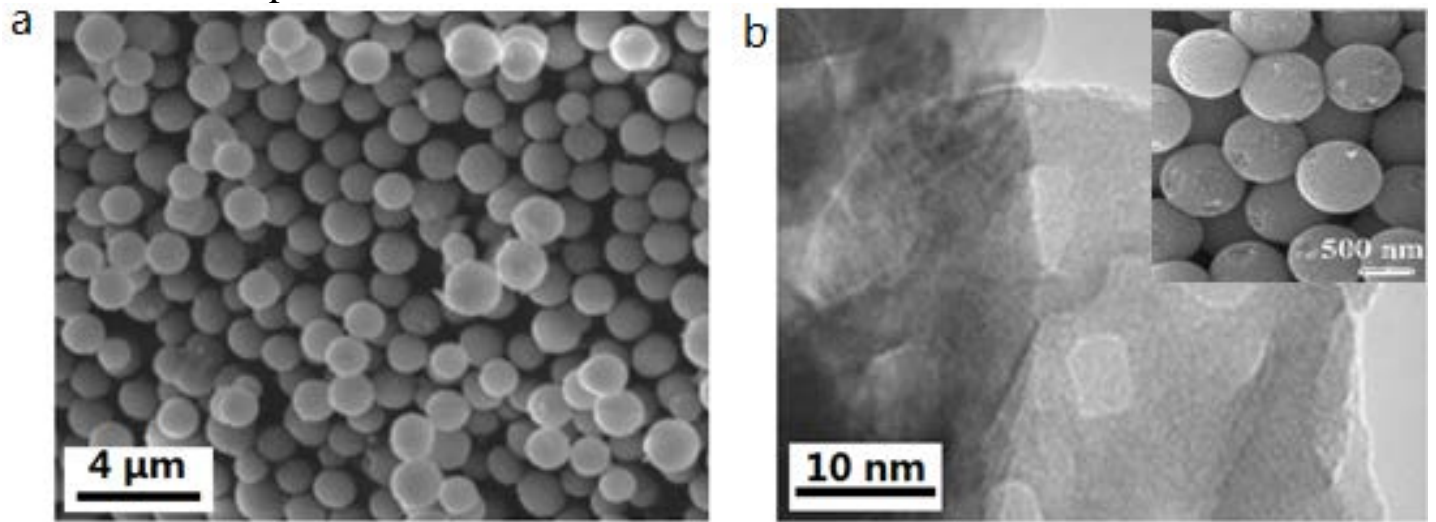

Fig.1. a. SEM, b. TEM image of the mesoporous $\mathrm{TiO}_{2}$ after calcination at $350^{\circ} \mathrm{C}$

The corresponding X-Ray Diffraction (XRD) pattern of the prepared precursor materials and the calcined mesoporous $\mathrm{TiO}_{2}$ was shown in Fig. 2, which was measured with Rigaku D/MAX 2500 XRD diffractometer. From the XRD data, the mesoporous titania consist of anatase with a small amount of brookite. The normalized integral intensities of anatase and brookite are $108 \pm 18$ and $6 \pm 1$ respectively. Calculated the crystalline part of mesoporous $\mathrm{TiO}_{2}$ is composed of $87 \pm 2 \%$ anatase and $13 \pm 2 \%$ brookite, according to the reference intensity ratios (RIRs) from Table 1 . The prepared precursor materials without calcined powder shows no diffraction peaks and only a weak, broad hump, which means that the precursor materials without calcined powder is $\mathrm{XRD}$-amorphous $\mathrm{TiO}_{2}$. The crystalline part of commercial P25 consist of rutile-anatase mixture and the UV100 is pure anatase. From the integral intensities obtained by profile fitting with respects to the RIRs, the crystalline part of P25 is $86 \pm 2 \%$ anatase and $14 \% \pm 2$ rutile, which agrees well with reference [7].
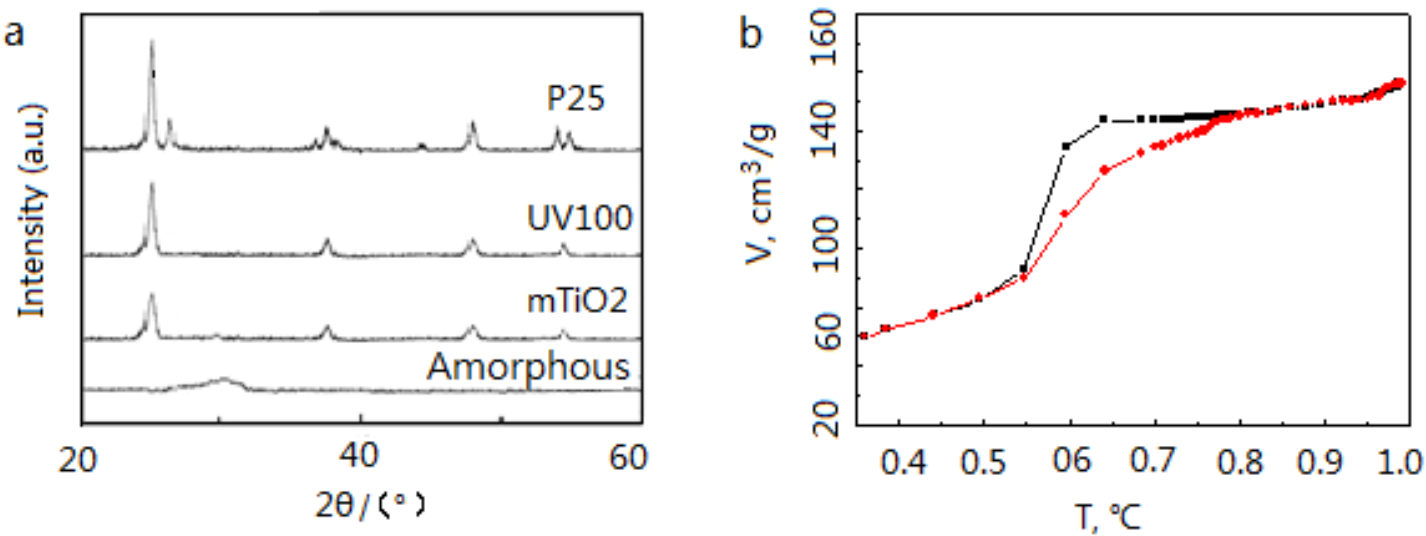

Fig. 2. a. XRD patterns of precursor material material (amorphous) and the calcined at $350^{\circ} \mathrm{C}$ mesoporous $\mathrm{TiO}_{2}$, b. Nitrogen sorption isotherms of the calcined mesoporous $\mathrm{TiO}_{2}$

It is necessary to measure samples' specific surface area (SSA) to determine their PCA. The nitrogen sorption isotherms for multipoint Brunauer-Emmett-Teller (BET) analysis and Barrett-Joyner-Halenda (BJH) calculations were obtained at $77 \mathrm{~K}$ using a Quantachrome Nova 2400e instrument, as shown in fig. 2 b. Before measurements, the samples were evacuated for $6 \mathrm{~h}$ at $200^{\circ} \mathrm{C}$. It was shown that the mesoporous titania has a SSA about $160 \mathrm{~m}^{2} / \mathrm{g}$, and the pore size distribution with a maximum pore radius of $4 \mathrm{~nm}$, which was in consistence with TEM.

Table 1. ICDD card numbers.

\begin{tabular}{|c|c|c|}
\hline phase & RIR & ICDD Card \\
\hline Silicon & $4.7 \pm 0.2$ & $27-1402,75-0589$ \\
\hline Anatase & $4.8 \pm 0.5$ & $71-1169,78-2486,84-1286$ \\
\hline Brookite & $1.6 \pm 0.3$ & $76-1934$ \\
\hline Rutile & $3.4 \pm 0.2$ & $21-1276,72-1148,73-1232$ \\
\hline
\end{tabular}

We added different weights of amorphous $\mathrm{TiO}_{2}$ with standard material, silicon powder, and 
measured the XRD data, as shown in fig. 3 a. The le Bail method of profile analysis was used for integral intensity measurements for the XRD patterns. And the composition of the crystalline component of amorphous phase $\omega_{\text {XRD }}\left(A_{i}\right)$ was calculated.

$$
X_{i}=\frac{I\left(A_{i}\right)}{\operatorname{RIR}_{i}} \quad \omega_{\text {KRD }}\left(A_{i}\right)=\frac{x_{A_{i}}}{\Sigma_{j} x_{j}}=100 \%
$$

In the formula, $I\left(A_{i}\right)$ is averaging the strong peak of each phase, $<\mathrm{hkl}>$, intensities and normalizing to the theoretical intensities. $\omega_{X R D}\left(A_{i}\right)$, corresponds to the percentage of the $A i$ phase in the crystalline portion of the mixture.

To determine the amorphous weight of the sample, we calculated with formula $3 . m_{\text {Sample }}$ is the real weight of the sample, $m_{S t}$ is crystalline standard amount, $\omega_{\text {real }}(S t)$ is corresponds to real weight percentage of the crystalline phase.

$$
\begin{aligned}
& \omega_{\text {Am }}=100 \% \times m_{\text {Am }} / m_{\text {total }} \\
& \omega_{\text {real }}(S t)=\frac{m_{\text {St }}}{m_{\text {St }}+m_{\text {Sample }}} \times 100 \% \\
& \omega_{\text {Am }}=100 \% \frac{m_{\text {Am }}}{m_{\text {Sample }}}=1+m_{S t} / m_{\text {Sample }} \times\left(1-1 / \omega_{\text {XRD }}(S t)\right)
\end{aligned}
$$
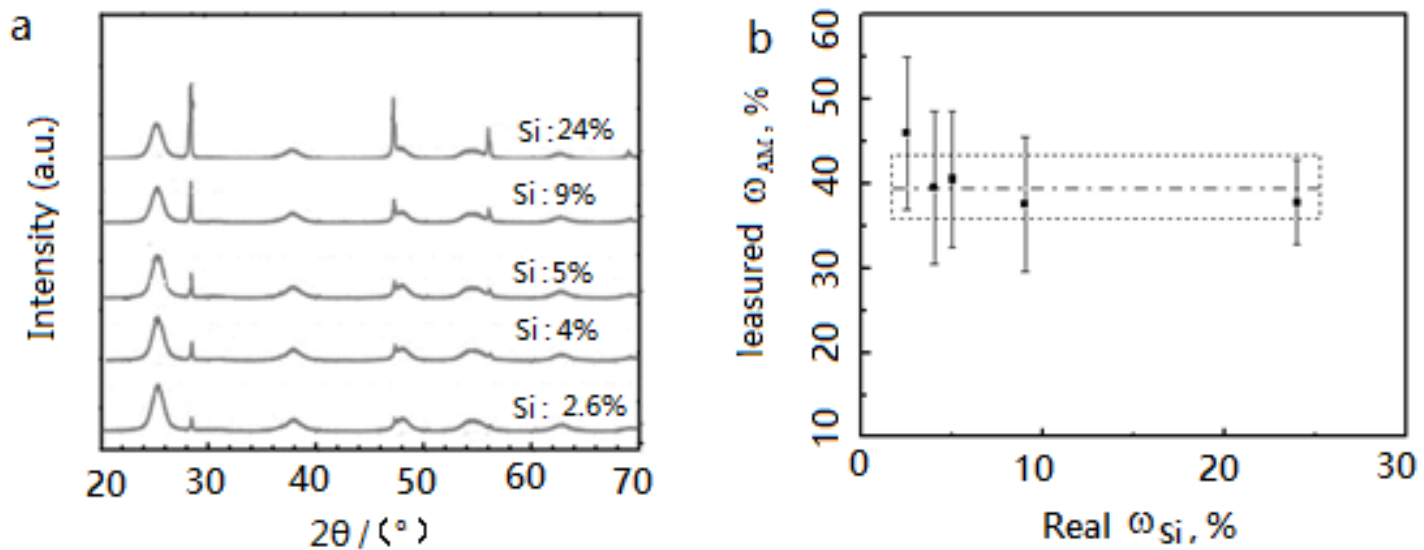

Fig. 3. a. XRD patterns of $\mathrm{Si}$ and $\mathrm{mTiO}_{2}$ sample mixture. b. Calculated $\omega_{A m}$ vs $\mathrm{Si}$ amount, mean value with deviation labeled with a straight line.

To reduce these errors and confirm the results, we measure every sample a few times with the addition of different amounts of the amorphous sample and crystalline standard. For this, we prepared five mixtures of each sample and the silicon standard powder, with Si weight percentages ranging from $2.6 \%$ to $50 \%$. Then the measured XRD patterns and was fitted using le Bail method to measure the intensities of the peaks for anatase and silicon. And based this, we the mean integral intensity of silicon for each mixture and calculated $\omega_{A m}$. Then we plotted the calculated $\omega_{A m}$ against $\omega_{S i}$, as shown in fig.3b. We can observe that all the data and check the slope coefficient, and they are within the error range. We average all of the $\omega_{A m}$ and calculate the standard deviation. From the fig. 3b, we can be sure of the consistency of this method. Data from other samples obtained by this method are shown in table 2 . 
Table 2 Crystalline phase composition, SSA, measured amount of amorphous phase and PCA. 。

\begin{tabular}{|c|c|c|c|c|}
\hline Sample & $\mathbf{R : ~ A : ~ B ~}$ & $\mathbf{S S A}, \mathbf{~ m}^{\mathbf{2}} \mathbf{g}$ & $\mathbf{P C A}_{\mathbf{m}}, \mathbf{\%}$ & $\boldsymbol{\omega}_{\mathbf{A m}}, \mathbf{\%}$ \\
\hline Amorphous $\mathrm{TiO}_{2}$ & $-:-:-$ & 250 & 0 & 100 \\
\hline P25 & $14: 86: - \pm 2$ & 40 & 100 & $14 \pm 1$ \\
\hline UV100 & $-: 100:-$ & 330 & 15 & $32 \pm 2$ \\
\hline UV100ac & $-: 100:-$ & 280 & 75 & $32 \pm 2$ \\
\hline UV100@400 & $-: 100:-$ & 170 & 45 & $25 \pm 2$ \\
\hline UV100@500 & $-: 100:-$ & 130 & 42 & $20 \pm 2$ \\
\hline $\mathrm{mTiO}_{2}$ & $-: 87: 13 \pm 3$ & 160 & 5 & $30 \pm 1$ \\
\hline $\mathrm{mTiO}_{2}$ ac & $-: 87: 13 \pm 3$ & 160 & 33 & $24 \pm 2$ \\
\hline $\mathrm{mTiO}_{2} @ 400$ & $-: 87: 13 \pm 5$ & 110 & 60 & $23 \pm 2$ \\
\hline $\mathrm{mTiO}_{2} @ 500$ & $-: 90: 10 \pm 5$ & 60 & 85 & $19 \pm 2$ \\
\hline
\end{tabular}

$\mathrm{R}: \mathrm{A}: \mathrm{B}$ - rutile: anatase: brookite weight ratio in crystalline portion $\mathrm{mTiO}_{2}$ is mesoporous $\mathrm{TiO}_{2}$ calcined at $350^{\circ} \mathrm{C}, \mathrm{mTiO}_{2}$ ac is removed amorphous titania with nitric acid, mTiO ${ }_{2} @ 400$ and $\mathrm{mTiO}_{2} @ 500$ are after annealed at $400^{\circ} \mathrm{C}$ and $500{ }^{\circ} \mathrm{C}$ mesoporous $\mathrm{TiO}_{2}$ 。

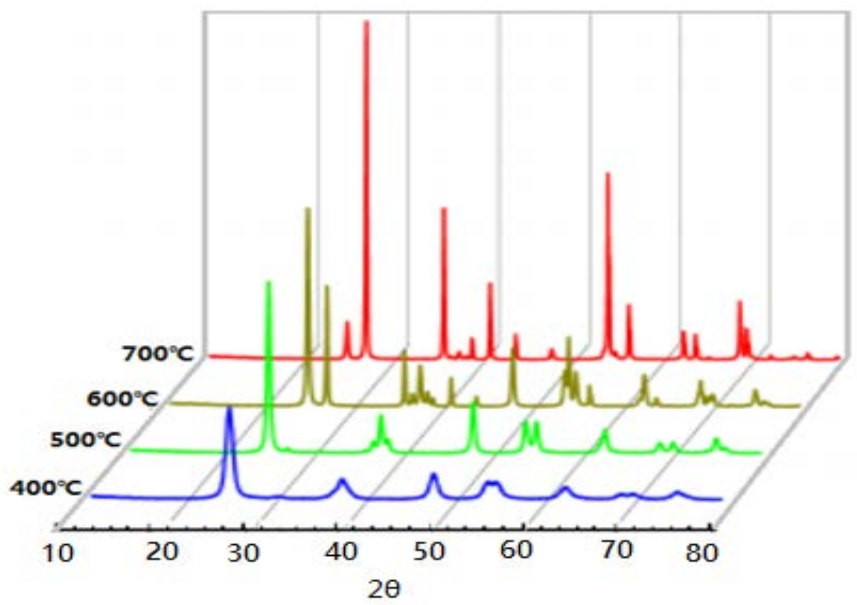

Fig. 4. XRD patterns of mesoporous $\mathrm{TiO}_{2}$ calcined with different temperature.

To remove the amorphous portion of $\mathrm{TiO}_{2}$, we can anneal the samples at temperatures higher than $400^{\circ} \mathrm{C}$. But during annealing, crystallization might be with phase transition and agglomeration of $\mathrm{TiO}_{2}$ particles with the decrease in SSA, which may significantly affect the PCA of the samples. So we performed fast annealing, the sample was placed in a hot oven for $15 \mathrm{~min}$. The rutile phase is observed in the samples obtained by fast heating to $600^{\circ} \mathrm{C}$, as shown in fig.4. Due to the impact of the phase composition on the PCA, we used only the samples annealed at $400^{\circ} \mathrm{C}$ and $500^{\circ} \mathrm{C}$.

Different titania phases with different dissolution rates in acids. So, based on the fast dissolution of amorphous titania in acid, we can selectively remove amorphous titania from samples by exposure to diluted nitric acid for a short time. A portion of the $\mathrm{TiO}_{2}$ sample was added to $1 \mathrm{M}$ HNO3 and stirred for 3 h, then washed with distilled water and dried at $60^{\circ} \mathrm{C}$ in an oven. The PCA of all samples were measured in the buffer solution ( $\mathrm{pH}$ 6.9), therefore, a small amount of residual acid should not change the measured PCA. Both annealing and acid method decrease the amorphous titania amount, and significantly increase the PCA of the samples, as shown in table 2 .

The PCA was measured through the decoloration of methyl orange (MO) in aqueous buffer solutions under high-pressure Hg bulb (5W) illumination. All measurements were performed at a temperature of $40^{\circ} \mathrm{C}$. 3mg of sample was used in each measurement, and the $\mathrm{TiO}_{2}$ concentration is $0.2 \mathrm{mg} / \mathrm{L}$ in the reaction mixture. The MO concentration in the mixture was $47 \mathrm{mg} / \mathrm{L}$. it is well known that the order of this reaction is pseudo-first in the case of both direct and indirect oxidation [8-11], therefore, decoloration rates were calculated by the linear approximation of the relative concentration of MO against time in a semi-log scale. To compare the measured rates of decoloration with the results of other groups, we defined the relative PCA of our samples by relating their MO decoloration rates of the samples $\left(\mathrm{r}_{\mathrm{S}}\right)$ to that of $\mathrm{P} 25\left(\mathrm{r}_{\mathrm{P} 25}=-0.0351 /(\mathrm{h} \mathrm{mg})\right.$ ) 
measured under the same conditions:

$$
\begin{aligned}
& P C A_{m}(S)=\frac{r_{S} / m_{S}}{r_{P_{25}} / m_{P_{25}}} \times 100 \% \\
& P C A_{S A}(S)=P C A_{m}(S) \cdot \frac{S S A_{P_{25}}}{S S A_{S}} \times 100 \%
\end{aligned}
$$

Where $P C A_{m}(S)$ is the PCA of the sample, normalized to the surface area. $P C A_{S A}(S)$ is normalized to the surface area. The PCA vs. $P C A_{m}(S)$ and PCA vs. $P C A_{S A}(S)$ for all crystallized samples was all plotted in fig.5.

In fig.5, it was demonstrated that all titania samples annealed at $400^{\circ} \mathrm{C}$ and $500^{\circ} \mathrm{C}$ or after acidification, the amorphous phase percentage were decreased, which leads to the PCA increased. In fig. 5.c, in curve UV100 sample, one can see that even small changes in amorphous content and SSA may result in strong increases of the PCA. This effect is probably due to changes in the spatial distribution of the amorphous phase [12]. Therefore, the influence of the amorphous phase spatial distribution on PCA should be investigated in the future.
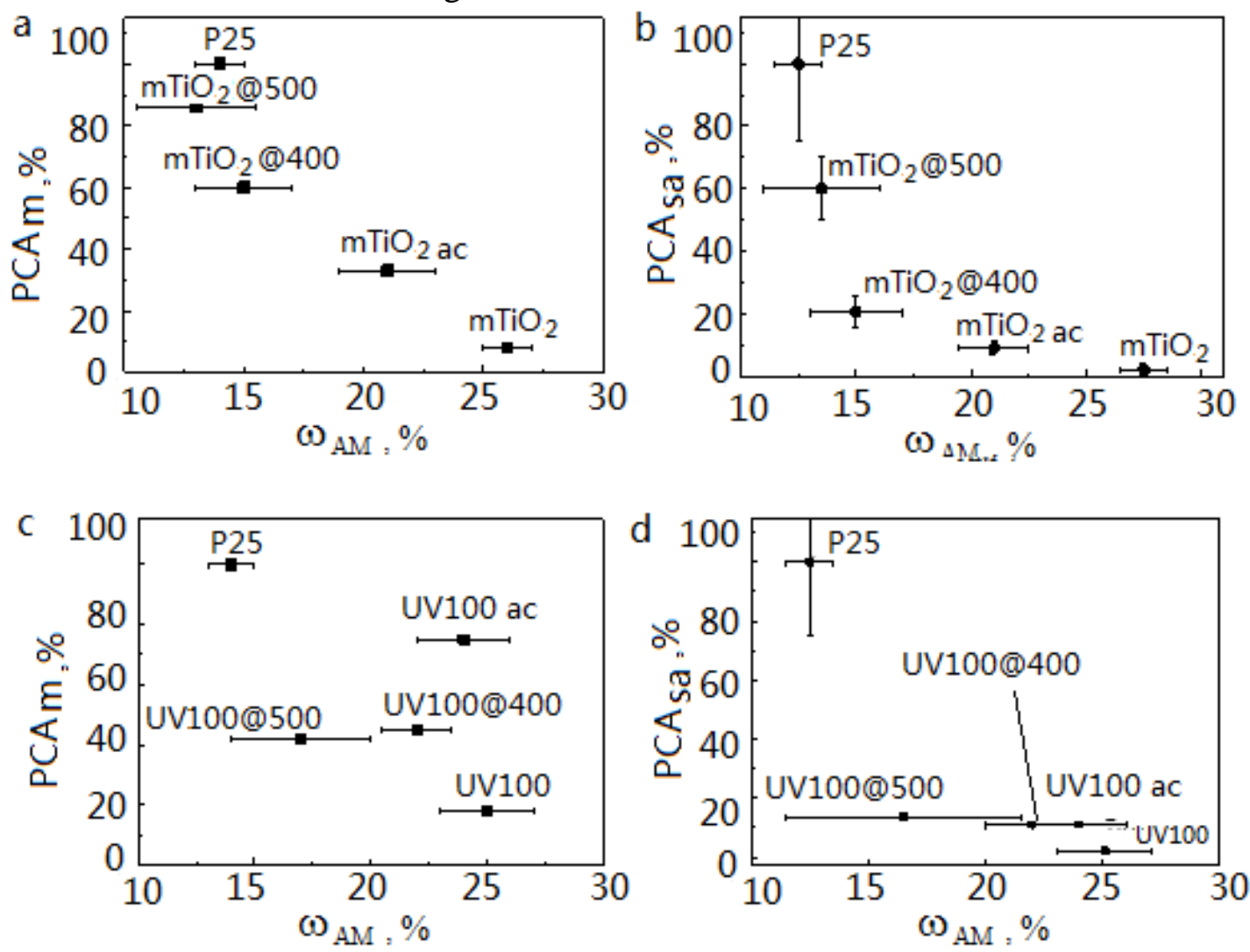

Fig. 5. a. Dependence of photocatalytic activity, normalized on the sample weight vs. amorphous titania amount, b. Dependence of photocatalytic activity, normalized on the sample surface area vs. amorphous titania amount of mesoporous $\mathrm{TiO}_{2}$ c. Dependence of photocatalytic activity, normalized on the sample weight vs. amorphous titania amount, d. Dependence of photocatalytic activity, normalized on the sample surface area vs. amorphous titania amount of mesoporous UV100.

\section{Conclusions}

In this work, we demonstrate that the presence of the amorphous $\mathrm{TiO}_{2}$ phase can directly affect the PCA. As measured, the samples containing a higher amount of the amorphous phase show a lower PCA. The proposed method for the quantitative analysis of the XRD-amorphous phase allows one to measure the weight percent of the amorphous titanium fraction with high accuracy. 


\section{Acknowledgement}

In this paper, the research was sponsored by the Natural Science Foundation of Jiangsu Province Department of Education (Project No. 16KJB430004).

\section{References}

[1] M. Kaneko, I. Okura, Photocatalysis: Science and Technology, Kodansha and Springer, New York, 2002.

[2] M.A. Henderson, A surface science perspective on photocatalysis [J], Surface Science Reports, 2011: 66(6-7): 185-297.

[3] M. Gratzel, Properties and Applications of Nanocrystalline Electronic Junctions, 2000.

[4] L. Saadoun, J. Ayllón, J. Jiménez-Becerril, J. Peral, X. Domènech, R. RodríguezClemente, 1,2-Diolates of titanium as suitable precursors for the preparation of photoactive high surface titania [J], Applied Catalysis B: Environmental, 1999: 21 (4): 269-277.

[5] H. Jensen, K. Joensen, J.-E. Jørgensen, J. Pedersen, G. Søgaard, Characterization of nanosized partly crystalline photocatalysts [J]. Journal of Nanoparticle Research, 2004: 6 (5): 519-526.

[6] J.M. Kim, Y.-J. Han, B.F. Chmelka, G.D. Stucky, One-step synthesis of ordered mesocomposites with non-ionic amphiphilic block copolymers: implications of isoelectric point, hydrolysis rate and fluoride, Chemical Communications [J]. 2000: 2437-2438.

[7] B. Ohtani, O. Prieto-Mahaney, D. Li, R. Abe, What is Degussa (Evonik) P25 Crystalline composition analysis, reconstruction from isolated pure particles and photocatalytic activity test, J. Photochem. Photobiol. A: Chem. [J] 2010: 216 (2-3): 179-182, 3rd International Conference on Semiconductor Photochemistry, SP-3, Glasgow, UK. April, 2010.

[8] N. Guettaï, H.A. Amar, Photocatalytic oxidation of methyl orange in presence of titanium dioxide in aqueous suspension. Part II: kinetics study, Desalination and the Environment [J] 2005: 185 (1-3): 439-448.

[9] D. Monllor-Satoca, R. Gómez, M. Gonzáez-Hidalgo, P. Salvador, The “Direct/Indirect” model: an alternative kinetic approach in heterogeneous photocatalysis based on the degree of interaction of dissolved pollutant species with the semiconductor surface [J], Catalysis Today, 2007: 129 (1-2): 247-255, Selected Contributions of the 4th European Meeting on Solar Chemistry and Photocatalysis: Environmental Applications (SPEA 4).

[10] Y. Li, X. Li, J. Li, J. Yin, Photocatalytic degradation of methyl orange by $\mathrm{TiO}_{2}$-coated activated carbon and kinetic study [J], Water Research, 2006: 40 (6) : 1119-1126.

[11] S. Al-Qaradawi, S.R. Salman, Photocatalytic degradation of methyl orange as a model compound, Journal of Photochemistry and Photobiology A: Chemistry, [J] 2002: 148 (1-3): 161-168.

[12] K. Tanaka, M.F. Capule, T. Hisanaga, Effect of crystallinity of $\mathrm{TiO}_{2}$ on its photocatalytic action [J], Chemical Physics Letters, 1991: 187 (1-2): 73-76. 